punto org

Collana diretta da Luigi Maria Sicca

57 


\title{
IMPRENDITORIALITÀ E SETTORE MUSEALE
}

\section{ESPERIENZE E PROSPETTIVE DI CAMBIAMENTO}

\author{
a cura di
}

\section{Maria Elena Santagati e Luca Zan}

Chiara Bombardieri - Paola Degli Esposti - Mariagabriella Fornasiero Valentina Galloni - Alessandro Gazzotti - Claudia Giordani - Federica Guidi Annalisa Managlia - Umberto Mossetti - Michele Recanatini

Elisa Schiavina - Stefania Spaggiari - Patrizia Tomba - Cinzia Vecchi 


\section{Indice}

11 Introduzione

Maria Elena Santagati e Luca Zan

\section{Parte prima \\ MUSEI DiVersi, SINTOMI COMUNI: \\ LA SINDROME CRONICA DELL'ESSERE MUSEO (IN ITALIA)}

1. Il Museo Archeologico Nazionale di Firenze Michele Recanatini

1.1. Nascita e breve storia

1.2. Assetto istituzionale

1.3. Risorse umane

1.4. Risorse finanziarie

1.5. Attività

1.5.1. Attività espositiva

29

1.5.2. Attività didattica

35

1.5.3. Visitatori e bigliettazione

38

1.5.4. Promozione e Comunicazione

39

1.6. Partnership

40

41

1.7. Prospettive di cambiamento

1 Bibliografia

2. Il Museo della ceramica di Fiorano Modenese Stefania Spaggiari

2.1. Nascita e breve storia

2.2. Assetto istituzionale 
59 2.6. Partnership

60 2.7. Prospettive di cambiamento

62 Bibliografia

\section{Parte seconda}

MUSEI CIVICI IN RETE: EQUILIBRIO INSTABILE TRA POLITICA, POLITICHE E BUROCRAZIA

67

67

70

74

76

77

78

79

81

81

87

87

89

92

92

95

96

96

97

102

102

104

3. Istituzione Bologna Musei

Claudia Giordani, Federica Guidi ed Elisa Schiavina

3.1. Nascita, breve storia e assetto istituzionale

3.2. Risorse umane (dal 2013 al 2017)

3.3. Risorse finanziarie

3.4. Attività

3.4.1. Attività didattiche

3.4.2. Le esposizioni temporanee

3.4.3. Valorizzazione e promozione

3.5. Partnership

3.6. Prospettive di cambiamento

4. I Musei civici di Reggio Emilia

Alessandro Gazzotti

4.1. Nascita e breve storia

4.2. Assetto istituzionale $1998-2018$

4.3. Risorse umane

4.3.1. Il personale interno ed esterno al Comune 4.3.2. Volontariato

4.4. Risorse finanziarie

4.4.1. Voci di entrata e di spesa relative all'anno 2014

4.4.2. Voci di entrata e di spesa relative all'anno 2017

4.5. Attività

4.5.1. Conservazione

4.5.2. Didattica 


\section{Parte terza \\ Musei UNiversitari tra PASSATO E FUtURo}

5. Il Museo di Geologia e Paleontologia dell'UniverSITÀ DEgLi StUdi di PAdova Mariagabriella Fornasiero 5.1. Nascita e breve storia 5.2. Assetto istituzionale

5.4. Risorse finanziarie

5.5. Attività

\subsubsection{Catalogazione}

5.5.2. Ricerca

5.5.3. Conservazione delle collezioni

5.5.4. Progettazione

5.5.5. Comunicazione scientifica

5.5.6. Partecipazione a network

5.6. Prospettive di cambiamento Bibliografia

\section{Sitografia}

6. L'Orto botanico dell'Alma Mater Studiorum UniVERsità di BolognA

Paola Degli Esposti e Annalisa Managlia con la collaborazione di Umberto Mossetti

6.1. Nascita e breve storia 
6.2. Assetto istituzionale

144 6.3. Risorse umane

144 6.4. Risorse finanziarie

6.5. Attività

\section{Parte quarta Musei "INVOLONTARI":}

IL PATRIMONIO CULTURALE DELLE ISTITUZIONI SANITARIE

7. Il Museo di Storia della psichiatria e il patrimonio storico dell'ex Ospedale psichiatrico San Lazzaro di Reggio Emilia

Chiara Bombardieri

161 7.1. Nascita e breve storia

164 7.2. Assetto istituzionale

166 7.3. Risorse umane

166 7.3.1. Biblioteca e archivio

167 7.3.2. Museo

$170 \quad$ 7.4. Risorse finanziarie

171 7.5. Attività

171

7.5.1. La biblioteca

173 7.5.2. Il museo

176 7.6. Partnership

178 7.7. Prospettive di sviluppo

180 Bibliografia 
183 8. Biblioteca Umberto I e Donazione Putti dell'Istituto Ortopedico Rizzoli di Bologna Patrizia Tomba

8.1. Nascita e breve storia

8.2. Assetto istituzionale

8.3. Risorse umane

8.4. Risorse finanziarie

8.5. Attività

\section{Parte quinta}

\section{Oltre la fruizione:}

VALORIZZARE EDUCANDO AL PATRIMONIO CULTURALE

9. "Io amo i beni culturali". Origine e sviluppo di UN PROGETTO REGIONALE

Valentina Galloni

197 9.1. Contesto e origine

200 9.2. Il Concorso

202 9.3. Risultati

206 9.4. Comunicazione

207 9.5. Risorse umane e finanziarie

208 9.6. Partner

209 9.7. Prospettive di sviluppo

213 10. Festival Francescano

Cinzia Vecchi

213 10.1. Nascita, breve storia e sviluppi futuri

220 10.2. Assetto istituzionale 
10.3. Risorse umane

223

10.4. Risorse finanziarie

223

10.4.1. Costi

224

10.4.2. Risorse

225

10.5. Attività

225 10.5.1. La programmazione

227 10.5.2. L'analisi dei visitatori

232

234 Bibliografia 10.6. Prospettive di cambiamento

237 Considerazioni conclusive

Maria Elena Santagati e Luca Zan

245 Notizie sugli autori

249 Hanno scritto nella Collana punto org 


\section{Hanno scritto nella Collana punto org}

www.puntoorg.net

1. L.M. Sicca (a cura di) ${ }^{1}$, Leggere e scrivere organizzazioni. Estetica, umanesimo e conoscenze manageriali (con postfazione di F. Piro), 2010.

2. L.M. Sicca, Alla fonte dei saperi manageriali. Il ruolo della musica nella ricerca per l'innovazione e per la formazione delle risorse umane, 2012.

3. A. Di Scipio, Pensare le tecnologie del suono e della musica (con prefazione di R. Diana), 2012.

4. R. Musto, Scienza Natura Cambiamento (con prefazione di M. Nicodemi), 2012.

5. R. Musto, Novalis. L'assoluto e le cose (con prefazione di C. Albarella e postfazione di G. Imbruglia), 2013.

6. Aa.Vv." I I linguaggi dell'organizzare. Musica e testo tra dono e disinteresse, 2013.

7. B. Masiello, Fiducia nelle reti. Strategie per la crescita nei mercati internazionali delle PMI (con prefazione di F. Izzo), 2013.

8. Aa.Vv."I, Tavola rotonda. Umanesimo del management attraverso gli occhi dell'altro, 2013.

9. M. Calcagno, Narrare terre di mezzo. Management arte design (con prefazione di S. Faccipieri e postfazione di A. Comacchio), 2013.

10. R. Diana, Disappartenenza dell'To. Filosofia e musica verso Samuel Beckett (con prefazione di L.M. Sicca), 2014.

${ }^{1}$ Con scritti di Per Olof Berg e Kristian Kreiner, Robert W. Witkin, Barbara Czarniawska e Carl Rhodes, Ken Starkey e Sue Tempest, John Hendry, Karin Knorr Cetina.

"Con scritti di Luigi Maria Sicca, Umberto di Porzio, Rosario Diana, Agostino

Di Scipio, Mariella De Simone, Bernardo Maria Sannino, Chiara Mallozzi, Lorenzo Pone, Giancarlo Turaccio.

"II Con scritti di Luigi Maria Sicca, Francesco Izzo, Maura Striano, Giulia Dell'Aquila, Felice Casucci, Francesco Perillo, Rosario Diana, Paola Giampaolo, Davide Bizjak, Gilberto-Antonio Marselli, Franco Vitelli, Maria Rosaria Napolitano. 
11. Aa.Vv." , Sergio Piro. Maestri e allievi, 2014.

12. F.D. Perillo (a cura di) ${ }^{\mathrm{v}}$, Impresa imperfetta, 2014.

13. L.M. Sicca, L. Zan (a cura di) ${ }^{\mathrm{v}}$, Management Arti Culture. Resoconto del primo anno del GSA - Accademia Italiana di Economia Aziendale, 2014.

14. M. Iaccarino, Un mondo assetato. Come il bisogno di acqua plasma la civiltà (con prefazione di F.P. Casavola e postfazione di A. Giannola), 2015.

15. F. Piro, Manuale di educazione al pensiero critico. Comprendere e argomentare (con prefazione di T. De Mauro), 2015.

16. F. D'Errico, Fuor di metafora. Sette osservazioni sull'improvvisazione musicale (con prefazione di P. de Vita e postfazione di M. Maldonato), 2015.

17. E. Mollona, Computer Simulation in Social Sciences. A Logic of Enquiry (with a foreword by G. Colombo and an afterword by D. Secchi), 2015.

18. S. Oliverio, L.M. Sicca, P. Valerio ${ }^{\mathrm{vI}}$, Transformare le pratiche nelle organizzazioni di lavoro e di pensiero (con prefazione di G. Manfredi), 2015.

19. P. Valerio, C. Bertolazzi, P. Marcasciano (a cura di) ${ }^{\text {vm, }}$, Transformare l'organizzazione dei luoghi di detenzione. Persone transgender e gender nonconforming tra diritti e identità (con prefazione di L.M. Sicca), 2016.

rv Con scritti di Giuseppe Cantillo, Tullio De Mauro, Aldo Masullo, Mariapaola Fimiani, Teresa Capacchione, Antonio Mancini, Roberto Beneduce, Enrico De Notaris, Fulvio Marone, Dario Stefano Dell'aquila, Luigi Maria Sicca, Francesco Piro.

v Con scritti di Pier Luigi Celli, Eugenio Mazzarella, Enzo Rullani, Luigi Maria Sicca, Francesco Varanini.

vi Con scritti di Stefano Baia Curioni, Paola Dubinie Ludovica Leone, Sara Bonini Baraldi e Luca Zan, Monica Calcagno e Luigi M. Sicca, Donata Collodi, Francesco Crisci e Andrea Moretti, Roberto Ferrari e Alessandro Hinna, Francesco Giaccari, Francesca Imperiale e Valentina Terlizzi, Daniele Goldoni, Pamela Palmi.

${ }^{v i}$ Con scritti di Anna Lisa Amodeo, Christian Ballarin, Davide Bizjak, Ilaria Boncori e Paolo Fazzari, Rossella Bonito Oliva, Simone Cangelosi, Marco De Giorgi, Guglielmo Faldetta, Vittoria Fiorelli, Stefano Maltese, Porpora Marcasciano, Piergiorgio Masi, Antonia Monopoli e Chiara Repetto, Andrea Morniroli, Edoardo Mollona, Cristiano Scandurra, Luca Solari, Maria Spanò, Maria Gigliola Toniollo.

${ }^{v i l}$ Con scritti di: Paolo Valerio, Giuseppe Ferraro, Carmen Bertolazzi, Alexander Hochdorn, Porpora Marcasciano, Luca Chianura, Damiana Massara, Daniela A. Nadalin, Adriana Godano, Luca Chianura, Vittoria Colonna, Elia De Caro, Tito Flagella, Anna Lorenzetti. 
20. M.R. Napolitano, V. Marino (a cura di) ${ }^{\mathrm{Ix}}$, Cultural Heritage e Made In Italy. Casi ed esperienze di marketing internazionale (con prefazione di G. Volpe e postfazione di A. Mattiacci), 2016.

21. M. Lusiani, Discourses of Planning (with a preface by L. Zan and an afterword by A. Langley), 2016.

22. F.D. Perillo, Simposio manageriale (con prefazione di A. Masullo e postfazione di P.L. Celli), 2016.

23. P. Ferri, I commissariamenti nel settore culturale italiano. Obiettivi, azioni, risultati (con prefazione di L. Zan e postfazione di G. Grossi), 2016.

24. L. Pareschi, Controcampo letterario. Strategie di intermediazione e accesso all'industria editoriale (con prefazione di P. Dubini e postfazione di G. Colombo), 2016.

25. G.-A. Marselli, Mondo contadino e azione meridionalista. L'esperienza del Gruppo Rossi-Doria a Portici (con prefazione di E. Mazzetti e postfazione di F. Vitelli), 2016.

26. F. Accardi ${ }^{x}$, Risk and Control Governance. A value-creation perspective (with a preface by A. De Nicola; an introduction by V. Atella and an afterword by S. Bozzolan), 2017.

27. I. Boncori (ed.) ${ }^{\mathrm{x}}, L G B T+$ Perspectives. The University of Essex Reader, (with a foreword by A. Forster), 2017.

28. A. Papa, “... Una cappella cavata dentro il monte...". Storia minima del complesso monastico di S. Lucia al Monte (con prefazione di L. D'Alessandro), 2017.

ix Con scritti di Loretta Battaglia, Giuseppe Bertoli, Roberta Biandolino, Michelle Bonera, Enrico Bonetti, Mauro Cavallone, Elena Cedrola, Marta Cerquetti, Maria Chiarvesio, Anna Codini, Emanuela Conti, Eleonora Di Maria, Barbara Francioni, Antonella Garofano, Francesco Izzo, Giulia Lanzilli, Gaetano Macario, Giulio Maggiore, Francesca Magno, Vittoria Marino, Barbara Masiello, Michela Matarazzo, Alberto Mattiacci, Marta Maria Montella, Fabio Musso, Maria Rosaria Napolitano, Alessandro Pagano, Tonino Pencarelli, Giovanna Pegan, Michele Quintano, Riccardo Resciniti, Marcello Risitano, Angelo Riviezzo, Savino Santovito, Elisabetta Savelli, Michele Simoni, Annarita Sorrentino, Raffaella Tabacco, Donata Vianelli.

${ }^{x}$ Con scritti di Roberto Rosato, Nicoletta Mincato, Carlo Nicoletti, Paolo De Paolis, Alessandro Salibra Bove.

х Con scritti di Alison J. Taylor-Lamb, Jamie Raines, Thomas Currid and Carl Chandra, Martin Harrison and Peter Martin, Rainer Shulze, Fleur Jeans and Teresa Eade, Tuesday Wats, Amy Anderson, Sco Lawley. 
29. R. Diana, L.M. Sicca, G. Turaccio ${ }^{\mathrm{xI}}$, Risonanze. Organizzazione, musica, scienze (con prefazione di A. Strati e postfazione di A. Solbiati), 2017.

30. F. D'Errico, Armonia funzionale e modalità. Rudimenti per l'improvvisazione a indirizzo jazzistico (con introduzione di F. Piro e prefazione di R. Grisley), 2017.

31. M. Calcagno, Interpreting Innovation. Design Creativity Art (con introduzione di F. Izzo; prefazione di A. Moretti e postfazione di J. Metelmann), 2017.

32. G. Balirano, Gardaí E Badfellas: The Discursive Construction of Organised Crime in the Irish Media, 2017.

33. M.C. Mason, A. Moretti, Tattoo Management. Mercati, attori, valore, 2017.

34. P. Testa, Innovazione del modello di business. Le dimensioni latenti nella letteratura di management (con prefazione di L. Cantone), 2017.

35. L. Massa, Viva 'o re! Municipio e dintorni (con introduzione di L.M. Sicca; prefazione di E. Borgonovi e postfazione di C. Mochi Sismondi), 2017.

36. F. Pavan, Memini. Piccole storie di storia della musica (con introduzione di E. Mazzarella; prefazione di R. Alessandrini e postfazione di V. Moroni), 2017.

37. C. Mallozzi, D. Tortora ${ }^{\mathrm{xII}}$, La bottega del suono. Mario Bertoncini. Maestri e allievi (con prefazione di M. Nicodemi e postfazione di L.M. Sicca), 2017.

38. G. Melis, Collaborazione e apprendimento nei processi di co-creazione di valore. Il caso delle destinazioni turistiche (con prefazione di M.R. Napolitano e postfazione di B. Argiolas), 2018.

39. G. Viglia, A.C. Invernizzi, Il ruolo dell'hubris nella gestione imprenditoriale (con prefazione di C. Mauri), 2018.

${ }^{x I I}$ Con scritti di Davide Bizjak, Dario Casillo, Rosario Diana, Umberto Di Porzio, Agostino Di Scipio, Chiara Mallozzi, Mario Nicodemi, Lorenzo Pone, Rosalba Quindici, Sonia Ritondale, Tommaso Rossi, Bernardo Maria Sannino, Luigi Maria Sicca, Cristian Sommaiuolo, Giancarlo Turaccio, Paolo Valerio.

x"I Con scritti di Mario Bertoncini, Davide Bizjak, Gianmario Borio, Pietro Cavallotti, Andrew Culver, Francesco D'Errico, Charles de Mestral, Michelangelo Lupone, Chiara Mallozzi, Alessandro Mastropietro, Mario Nicodemi, Luigino Pizzaleo, Lorenzo Pone, Ingrid Pustijanac, John Rea, Bernardo Maria Sannino, Luigi Maria Sicca, Daniela Tortora. 
40. T. Russo Spena, C. Mele, Practising innovation. A Sociomaterial View (with a foreword by Evert Gummesson; preface by Jim Spohrer and an afterword by Paolo Stampacchia), 2018.

41. I. Boncori, Race, Ethnicity and Inclusion. The University of Essex Reader (with a foreword by A. Forster and a postface by M. Śliwa), 2018.

42. K.E. Russo, The Evaluation of Risk in Institutional and Newspaper Discourse. The Case of Climate Change and Migration (with a preface by G. Bettini), 2018.

43. R. Pera, When Consumers get Creative. Cocreation in the Individual and Collective Realm (with a preface by D. Dalli), 2018.

44. F. Piro, L.M. Sicca, P. Maturi, M. Squillante, M. Striano ${ }^{\mathrm{xIV}}$ (a cura di), Sfide didattiche. Il pensiero critico nella scuola e nell'università, 2018.

45. R. Quaglia, Bravi ma basta! Su certe premesse, promesse e catastrofi culturali (con introduzione di L.M. Sicca; prefazione di J. Mills e postfazione di F. Barca), 2018.

46. B. Czarniawska ${ }^{\mathrm{xv}}$, La narrazione nelle scienze sociali, 2018.

47. F. Longobardi, Le affinità del lessico, 2018.

48. G. Calogero ${ }^{\mathrm{xv1}}$, L'abbiccì della democrazia. E altri scritti, 2018.

49. V. Fiorelli (a cura di) ${ }^{\mathrm{xv}}$, Margini e confini. Attraversamenti di metodi e linguaggi tra comunicazione, didattica e possibilità della ricerca (con prefazione di L. d'Alessandro), 2018.

50. G. Cundari, Il mondo: una bella prigione? Riflessioni geografiche, 2018.

51. M. Nicotra, Il canto dei sireni. Invenzioni trans/singolari e psicoanalisi lacaniana (con prefazione di A. Vicens e postfazione di P. Guazzo), 2019.

xiv Con scritti di Maura Striano, Rosaria Capobianco e Maria Rita Petitti, Francesco Piro, Roberta Gimigliano, Monica Mollo, Gerarda Fattoruso, Maria Incoronata Fredella, Maria Grazia Olivieri, Massimo Squillante e Antonia Travaglione, Pietro Maturi, Fabio Maria Risolo, Luca Marano, Luigi Maria Sicca, Giuseppe Recinto, Mario Nicodemi, Chiara Mallozzi e Luigi Marolda, Luigi Proserpio, Davide Bizjak, Paolo Canonico, Stefano Consiglio, Ernesto De Nito e Teresa Anna Rita Gentile, Natascia Villani.

xv I edizione italiana a cura di Luigi Maria Sicca, Francesco Piro, Ilaria Boncori.

${ }^{x v 1}$ A cura di Renato Trombelli, con una testimonianza di Gennaro Sasso.

xvi Con scritti di Giuliano Amato, Gianluca Bocchi, Massimo Abdallah Cozzolino, Diego Davide, Lucia Donsì, Amedeo Feniello, Vittoria Fiorelli, Luigi Manconi, Roberta Morosini, Gianmarco Pisa, Ciro Pizzo, Leopoldo Repola, Stefano Rodotà, Francesco Varanini. 
52. L. Marano, Come parlano i giovani. Una indagine di sociolinguistica urbana (con prefazione di P. Maturi), 2019.

53. M. Floris, R. Tronci, C. Dessì, A. Dettori, Imprese familiari e imprenditorialità. La sfida del cambiamento (con prefazione di F. Cabiddu), 2019.

54. T.T. Lennerfors, L. Mitchell ${ }^{\mathrm{xv} I I}$ (eds), SCOS. Searching Collectively for Our Soul, 2019.

55. Aa.Vv. ${ }^{\mathrm{xix}}$, Lucio Sicca. Maestri e allievi, in corso di pubblicazione.

56. E. Sacerdote, Breviario sul pensiero strategico. Discorsi e percorsi per conquistare il futuro migliore, 2019.

xvm Con scritti di Beatriz Acevedo, Omar Aktouf, Noortje van Amsterdam, Per Olof Berg, Davide Bizjak, Ilaria Boncori, Jo Brewis, Peter Case, Patricia Ehrensal, Ken Ehrensal, Hugo Gaggiotti, Silvia Gherardi, Claes Gustafsson, Campbell Jones, Nina Kivinen, Monika Kostera, Kristian J Kreiner, Thomas Taro Lennerfors, Marcus Lindahl, Steve Linstead, Tomek Ludwicki, Takashi Majima, Laura Mitchell, Albert J. Mills, Jean Helms Mills, Luc Peters, Alf Rehn, Harriet Shortt, Luigi Maria Sicca, Antonio Strati, Sam Warren, Robert Witkin, Anthony R. Yue, Peter Zackariasson.

${ }^{x x}$ Con scritti di Luigi Guatri, Giovanni Zanetti, Adriano Giannola, Franco Amatori, Renato Mele, Francesco Testa e Riccardo Mercurio; Enzo Rullani, Paolo de Vita, Salvatore Vicari, Luigi Cantone, Pierpaolo Testa e Vincenzo Basile; Marco Ferretti, Maria Rosaria Napolitano, Francesco Izzo, Enrico Bonetti, Antonio Capaldo, Paolo Calvosa. 